\title{
INTERSECTION COHOMOLOGY OF A RANK ONE LOCAL SYSTEM ON THE COMPLEMENT OF A HYPERPLANE-LIKE DIVISOR
}

\author{
D. ARINKIN ${ }^{\dagger}$, A. VARCHENKO ${ }^{\diamond}$ \\ Department of Mathematics, University of North Carolina at Chapel Hill \\ Chapel Hill, NC 27599-3250, USA
}

\begin{abstract}
Under a certain condition A we give a construction to calculate the intersection cohomology of a rank one local system on the complement to a hyperplane-like divisor.
\end{abstract}

Let $X$ be a smooth connected complex manifold and $D$ a divisor. The divisor $D$ is hyperplane-like if $X$ can be covered by coordinate charts such that in each chart $D$ is the union of hyperplanes. Such charts are called linearizing.

Let $D$ be a hyperplane-like divisor, $V$ a linearizing chart. A local edge of $D$ in $V$ is any nonempty irreducible intersection in $V$ of hyperplanes of $D$ in $V$. A local edge is dense if the subarrangement of all hyperplanes in $V$ containing the edge is irreducible: the hyperplanes cannot be partitioned into nonempty sets so that, after a change of coordinates, hyperplanes in different sets are in different coordinates. An edge of $D$ is the maximal analytic continuation in $X$ of a local edge. Any edge is an immersed submanifold in $X$. An edge is called dense if it is locally dense.

Let $U=X-D$ be the complement to $D$. Let $\mathcal{L}$ be a rank one local system on $U$ with nontrivial monodromy around each irreducible component of $D$. We want to calculate the intersection cohomology $H^{*}\left(X ; j_{!_{*}} \mathcal{L}\right)$ where $j: U \rightarrow X$ is the embedding. In this calculation a role will be played by the dual local system $\mathcal{L}^{\vee}$ on $U$ with the inverse monodromy.

Let $\pi: \tilde{X} \rightarrow X$ be a resolution of singularities of $D$ in $X$.

Remark. Note that among the resolutions of singularities there is the minimal one. The minimal resolution is constructed by first blowing-up dense vertices of $D$, then by blowing-up the proper preimages of dense one-dimensional edges of $D$ and so on, see [V, STV].

The preimage $\pi^{-1}(D)$ is a hyperplane-like divisor in $\tilde{X}$. The inverse image $\pi^{*} \mathcal{L}$ is a rank one local system on the complement $\tilde{X}-\pi^{-1}(D)$. However, its monodromy around some components of $\pi^{-1}(D)$ may be trivial. Denote by $\tilde{D} \subset \pi^{-1}(D) \subset \tilde{X}$ the maximal divisor in $\tilde{X}$ such that $\pi^{*} \mathcal{L}$ has nontrivial monodromy around $\tilde{D}$. The local systems $\pi^{*} \mathcal{L}$ and $\pi^{*} \mathcal{L}^{\vee}$ extend to local systems on $\tilde{U}=\tilde{X}-\tilde{D}$ denoted by $\tilde{\mathcal{L}}$ and $\tilde{\mathcal{L}}^{\vee}$, respectively. For $x \in X$, denote $\tilde{U}_{x}=\pi^{-1}(x) \cap \tilde{U}$.

Definition 1. We say that the local system $\mathcal{L}$ on $U$ satisfies condition $A$ with respect to the resolution of singularities $\pi: \tilde{X} \rightarrow X$, if for any edge $F$ of $D$ and any point $x$ of $F$ that is not contained in any smaller edge, we have $H^{\ell}\left(\tilde{U}_{x} ;\left.\tilde{\mathcal{L}}\right|_{\tilde{U}_{x}}\right)=0$ for $\ell>$ codimF -1 . Similarly, we say that the local system $\mathcal{L}^{\vee}$ on $U$ satisfies condition $A$ with respect to the resolution of

${ }^{\dagger}$ Supported in part by the Sloan Fellowship

$\diamond$ Supported in part by NSF grant DMS-1101508 
singularities $\pi: \tilde{X} \rightarrow X$, if for any edge $F$ of $D$ and any point $x \in F$ that is not contained in any smaller edge, we have $H^{\ell}\left(\tilde{U}_{x} ;\left.\tilde{\mathcal{L}}^{\vee}\right|_{\tilde{U}_{x}}\right)=0$ for $\ell>\operatorname{codim} F-1$.

It is not hard to check that to verify condition A, it suffices to consider only dense edges $F$.

Notice that if the monodromy of $\mathcal{L}$ lies in $\left\{z \in \mathbb{C}^{\times}|| z \mid=1\right\}$ (in other words, $\mathcal{L}$ is a unitary local system), then $\mathcal{L}$ satisfies condition $\mathrm{A}$ with respect to $\pi$ if and only if $\mathcal{L}^{\vee}$ satisfies condition A with respect to $\pi$.

Theorem 1. If both local systems $\mathcal{L}$ and $\mathcal{L}^{\vee}$ on the complement $U$ to the hyperplane-like divisor $D$ in $X$ satisfy condition $A$ with respect to a resolution of singularities $\pi: \tilde{X} \rightarrow X$, then the intersection cohomology $H^{*}\left(X ; j_{!_{*}} \mathcal{L}\right)$ is naturally isomorphic to $H^{*}(\tilde{U} ; \tilde{\mathcal{L}})$.

Proof. Let $\tilde{j}: \tilde{U} \rightarrow \tilde{X}$ be the open embedding. Since $\tilde{\mathcal{L}}$ has non-trivial monodromy around every component of $\tilde{D}$ and $\tilde{D}$ has normal crossings, we have $\tilde{j}_{*} \tilde{\mathcal{L}}=\tilde{j}_{!} \tilde{\mathcal{L}}=\tilde{j}_{! *} \tilde{\mathcal{L}}$ as well as $\tilde{j}_{*} \tilde{\mathcal{L}}^{\vee}=\tilde{j}_{!} \tilde{\mathcal{L}}^{\vee}=\tilde{j}_{! *} \tilde{\mathcal{L}}^{\vee}$. We also have $H^{*}\left(X ; \pi_{*} \tilde{j}_{*} \tilde{\mathcal{L}}\right)=H^{*}(\tilde{U} ; \tilde{\mathcal{L}})$ and $H^{*}\left(X ; \pi_{*} \tilde{j}_{*} \tilde{\mathcal{L}}^{\vee}\right)=$ $H^{*}\left(\tilde{U} ; \tilde{\mathcal{L}}^{\vee}\right)$. To simplify notation, we write $\pi_{*}$ for the direct image in the derived category $R \pi_{*}$. Thus, $\pi_{*} \tilde{j}_{*} \tilde{\mathcal{L}}$ is a complex of sheaves (more precisely, an object of the corresponding derived category), and $H^{*}\left(X ; \pi_{*} \tilde{j}_{*} \tilde{\mathcal{L}}\right)$ is its hypercohomology.

The restriction of $\pi_{*} \tilde{j}_{*} \tilde{\mathcal{L}}$ to $U$ is $\mathcal{L}$. The theorem follows from the next lemma.

Lemma. Let $\pi: \tilde{X} \rightarrow X$ be a proper holomorphic map of complex manifolds. For $x \in X$, denote $\tilde{i}_{x}: \pi^{-1}(x) \rightarrow \tilde{X}$ the embedding of the fiber. Let $\tilde{\mathcal{F}}$ be a complex of sheaves with constructible cohomology sheaves on $\tilde{X}$. (In most examples, $\tilde{\mathcal{F}}$ is a perverse sheaf, see [BBD] for the definition.) Set $\tilde{\mathcal{F}}^{\vee}=\mathbb{D} \tilde{\mathcal{F}}$, where $\mathbb{D}$ is the Verdier duality functor.

Suppose $\tilde{\mathcal{F}}$ satisfies the following condition $B$ : for every $\ell>0$ there is an analytic subset $X_{\ell} \subset X$, codim $X_{\ell}=\ell+1$, such that for any $x \in X-X_{\ell}$ we have $H^{i}\left(\pi^{-1}(x) ; \tilde{i}_{x}^{*} \tilde{\mathcal{F}}\right)=0$ and $H^{i}\left(\pi^{-1}(x) ; \tilde{i}_{x}^{*} \tilde{\mathcal{F}}^{\vee}\right)=0$ for all $i \geqslant \ell$ or $i<0$. Then $\pi_{*} \tilde{\mathcal{F}}=\pi_{!} \tilde{\mathcal{F}}$ is a perverse IC-sheaf on $X$.

Proof. This lemma is a slight generalization of the results of Goresky and MacPherson ([GM, Section 6.2]) about small maps. The argument of [GM] applies without change. Indeed, by GM, Second theorem of Section 6.1], we need to verify that the complex $\pi_{*} \tilde{\mathcal{F}}$ is such that $H^{i}\left(\pi_{*} \tilde{\mathcal{F}}\right)=0$ for $i<0$ and $\operatorname{codim} \operatorname{supp}\left(H^{i}\left(\pi_{*} \tilde{\mathcal{F}}\right)\right)>i$ for $i>0$, and that the same is true for the dual complex $\mathbb{D} \pi_{*} \tilde{\mathcal{F}}=\pi_{*} \tilde{\mathcal{F}}^{\vee}$. But these conditions are clear by base change. (Note that unlike [GM], we use the non-self-dual normalization: for instance, a local system on a smooth manifold is an IC-sheaf in our convention, but it requires cohomological shift in the self-dual normalization.)

In particular, in the settings of the theorem, the lemma applies to $\mathcal{F}=\tilde{j}{ }_{*} \tilde{\mathcal{L}}$, because both $\mathcal{L}$ and $\mathcal{L}^{\vee}$ satisfy condition $\mathrm{A}$. This concludes the proof of the theorem.

Corollary. If the local systems $\mathcal{L}$ and $\mathcal{L}^{\vee}$ satisfy condition $A$ with respect to each of two resolutions of singularities $\pi: \tilde{X} \rightarrow X$ and $\pi^{\prime}: \tilde{X}^{\prime} \rightarrow X$, then $H^{*}(\tilde{U} ; \tilde{\mathcal{L}})$ and $H^{*}\left(\tilde{U}^{\prime} ; \tilde{\mathcal{L}}^{\prime}\right)$ are canonically isomorphic.

Example 1. Let $X$ be the projective space of dimension $k$. Let $D \subset X$ be the union of hyperplanes and $\mathcal{L}$ a rank one local system on $U=X-D$ with nontrivial monodromy around each hyperplane. Assume that $D$ has normal intersections at all edges except at 
vertices. Then both $\mathcal{L}$ and $\mathcal{L}^{\vee}$ satisfy condition $\mathrm{A}$ with respect to the minimal resolution of singularities. Indeed, to obtain $\tilde{X}$ one has to blow-up dense vertices of $D$. If $x \in D$ is a dense vertex, then $\tilde{U}_{x}$ is nonempty only if $\mathcal{L}$ has trivial monodromy across $x$. In that case $\tilde{U}_{x}$ is an affine variety of dimension $k-1$ and $H^{\ell}\left(\tilde{U_{x}} ;\left.\tilde{\mathcal{L}}\right|_{\tilde{U}_{x}}\right)=0, H^{\ell}\left(\tilde{U}_{x} ;\left.\tilde{\mathcal{L}}^{\vee}\right|_{\tilde{U}_{x}}\right)=0$ for $\ell \geqslant k$.

Example 2. Let $X$ be $\mathbb{C}^{3}$. Let $\mathcal{C}$ be the central arrangement of six planes

$$
\begin{array}{ll}
H_{1}: x_{1}-x_{3}=0, & H_{2}: x_{1}+x_{3}=0, \\
H_{3}: x_{2}-x_{3}=0, & H_{4}: x_{2}+x_{3}=0, \\
H_{5}: x_{1}-x_{2}=0, & H_{6}: x_{1}+x_{2}=0
\end{array}
$$

with weights $a_{1}=a_{2}, \quad a_{3}=a_{4}, \quad a_{5}=a_{6}, \quad a_{1}+a_{3}+a_{5}=0$. Let $\mathcal{L}$ be the local system on $U$ with the monodromy $e^{2 \pi i a_{j}} \neq 1$ around $H_{j}$. Let $\pi: \tilde{X} \rightarrow X$ be the minimal resolution of singularities. For $x=(0,0,0)$, the space $\tilde{U}_{x}$ is the projective plane with four blown-up points and six lines removed. We have $\operatorname{dim} H^{3}\left(\tilde{U}_{x}, \tilde{\mathcal{L}}\right)=1$. This weighted arrangement $\mathcal{C}$ does not satisfies condition $\mathrm{A}$ with respect to the minimal resolution of singularities.

Remark. If $D \subset \mathbb{C}^{k}$ is the union of hyperplanes of a central arrangement and the monodromy of $\mathcal{L}$ is close to 1 , then the intersection cohomology $H^{*}\left(X ; j_{! *} \mathcal{L}\right)$ was computed in [KV] as the cohomology of the complex of flag forms of the arrangement.

The following equivariant version of Theorem 1 holds. Let $G$ be a finite group, $\rho$ an irreducible representation of $G$. For a representation $M$ denote by $M^{\rho} \subset M$ the $\rho$-isotypical component.

Let $\pi: \tilde{X} \rightarrow X$ be a resolution of singularities of $D$ as before. Assume that $G$ acts on $X$ and $\tilde{X}$ so that the actions preserve $U, \mathcal{L}, \tilde{U}, \tilde{\mathcal{L}}$ and commute with the map $\pi$. Then $G$ acts on $H^{*}\left(X ; j_{! *} \mathcal{L}\right)$ and $H^{*}(\tilde{U} ; \tilde{\mathcal{L}})$.

For $x \in X$, we denote by $O_{x}$ the $G$-orbit of $x$.

Definition 2. We say that the local system $\mathcal{L}$ satisfies condition $A$ with respect to $\rho$ and a resolution of singularities $\pi$ if for any edge $F$ of $D$ and any point $x \in F$ that is not contained in any smaller edge, we have $H^{\ell}\left(\cup_{y \in O_{x}} \tilde{U}_{y} ;\left.\tilde{\mathcal{L}}\right|_{\cup_{y} \in O_{x}} \tilde{U}_{y}\right)^{\rho}=0$ for $\ell>$ codimF -1 . Similarly, we say that the local system $\mathcal{L}^{\vee}$ on $U$ satisfies condition $A$ with respect to $\rho$ and a resolution of singularities $\pi$ if for any edge $F$ of $D$ and any point $x \in F$ that is not contained in any smaller edge, we have $H^{\ell}\left(\cup_{y \in O_{x}} \tilde{U}_{y} ;\left.\tilde{\mathcal{L}}^{\vee}\right|_{\cup_{y \in O_{x}} \tilde{U}_{y}}\right)^{\rho}=0$ for $\ell>\operatorname{codim} F-1$.

Generally speaking, we can no longer consider dense edges only in the equivariant version of condition A. In principle, in the equivariant case, for each edge $F$, it suffices to check only the generic points $x \in F$, even though the stabilizer might be different for other points.

Theorem 2. If both local systems $\mathcal{L}$ and $\mathcal{L}^{\vee}$ on $U$ satisfy condition $A$ with respect $\rho$ and a resolution of singularities $\pi$, then the intersection cohomology $H^{*}\left(X ; j_{!_{*}} \mathcal{L}\right)^{\rho}$ is naturally isomorphic to $H^{*}(\tilde{U} ; \tilde{\mathcal{L}})^{\rho}$ as $G$-modules.

Proof. Consider the quotient $X / G$, which may be singular. The quotient map $q: X \rightarrow X / G$ is finite; therefore, the derived direct image $q_{*}\left(j_{!_{*}} \mathcal{L}\right)$ is a perverse IC-sheaf on $X / G$. The direct image carries a fiber-wise action of $G$, so we can take the $\rho$-isotypical component, which is a direct summand $q_{*}\left(j_{!_{*}} \mathcal{L}\right)^{\rho} \subset q_{*}\left(j_{!_{*}} \mathcal{L}\right)$. Thus $q_{*}\left(j_{!_{*}} \mathcal{L}\right)^{\rho}$ is itself an IC-sheaf. 
Similarly, there is a direct summand $\left(q_{*} \pi_{*} \tilde{j}_{*} \tilde{\mathcal{L}}\right)^{\rho} \subset q_{*} \pi_{*} \tilde{j}_{*} \tilde{\mathcal{L}}$. By the argument used in the proof of Theorem $1,\left(q_{*} \pi_{*} \tilde{j}_{*} \tilde{\mathcal{L}}\right)^{\rho}$ is a perverse IC-sheaf. It is thus identified with $q_{*}\left(j_{! *} \mathcal{L}\right)^{\rho}$.

The authors thank A. Levin and M. Falk for helping develop Example 2.

\section{REFERENCES}

[BBD] A. Beilinson, J. Bernstein, P. Deligne, Faisceaux pervers, Astrisque 100, Paris, Soc. Math. Fr. 1982

[GM] M. Goresky, R. MacPherson, Intersection homology. II, Invent. Math. 72(1983), no. 1, 77-129.

[KV] S. Khoroshkin, A. Varchenko, Quiver D-modules and homology of local systems over an arrangement of hyperplanes, IMRP, 2006, doi: 10.1155

[STV] V.Schechtman, H. Terao, A. Varchenko, Local systems over complements of hyperplanes and the Kac-Kazhdan conditions for singular vectors, J. Pure Appl. Algebra 100 (1995), 93-102

[V] A. Varchenko, Multidimensional hypergeometric functions and representation theory of Lie algebras and quantum groups, Advances in Math. Phys., 21, World Scientific, 1995 